\title{
Imaging of hepatocellular carcinoma and image guided therapies - how we do it
}

\author{
Jonathon Willatt ${ }^{*}$ D, Julie A. Ruma, Shadi F. Azar, Nara L. Dasika and F. Syed
}

\begin{abstract}
Treatment options for hepatocellular carcinoma have evolved over recent years. Interventional radiologists and surgeons can offer curative treatments for early stage tumours, and locoregional therapies can be provided resulting in longer survival times. Early diagnosis with screening ultrasound is the key. CT and MRI are used to characterize lesions and determine the extent of tumour burden. Imaging techniques are discussed in this article as the correct imaging protocols are essential to optimise successful detection and characterisation. After treatment it is important to establish regular imaging follow up with $\mathrm{CT}$ or MRI as local residual disease can be easily treated, and recurrence elsewhere in the liver is common.
\end{abstract}

\section{Background}

Hepatocellular carcinoma (HCC) is the most common liver cancer and the fifth most common cancer worldwide. It results in between 250,000 and 1 million deaths globally per annum [1]. The number of deaths per year in HCC is close to that of the incidence throughout the world, which emphasizes the high case fatality rate of this aggressive cancer [1].

$80 \%$ of HCC cases are associated with chronic hepatitis $\mathrm{B}$ and $\mathrm{C}$ virus infections [2]. Alcoholic liver disease is a risk factor in younger age groups, and the combination of alcoholic liver disease and viral hepatitis substantially increases the risk for the development of cirrhosis and HCC. The obesity epidemic has resulted in a growing population of patients with non-alcoholic fatty liver disease, cirrhosis and HCC [3].

In the United States, HCC, with its link to the hepatitis $\mathrm{C}$ epidemic, represents the fastest growing cause of cancer mortality overall and the second fastest growing cause of cancer deaths among women [4].

\section{Surveillance}

The AASLD (American Association for the Study of Liver Diseases) recommends screening for the following high-risk groups: Asian male hepatitis B carriers over age 40, Asian female hepatitis B carriers over age 50, hepatitis B carriers with a family history of $\mathrm{HCC}$, Africans and

* Correspondence: jwillatt@med.umich.edu

Veterans Administration, University of Michigan, Ann Arbor, MI, USA
African Americans with hepatitis B, cirrhotic hepatitis B carriers, individuals with hepatitis $\mathrm{C}$ cirrhosis, individuals with stage 4 primary biliary cirrhosis, individuals with genetic hemochromatosis and cirrhosis, individuals with alpha 1-antitypsin deficiency and cirrhosis, individuals with cirrhosis from other etiologies [5].

We scan patients with cirrhosis from any etiology every 6 months with ultrasound $[5,6]$. Ultrasonography remains the primary imaging modality of choice for HCC surveillance. It is more cost-effective than CT and MRI, and more widely available. A meta-analysis reported a sensitivity of $94 \%$ in detecting lesions and a specificity of $>90 \%$ [7], although the figures were less favourable for lesions measuring less than $2 \mathrm{~cm}$. The sensitivity for early HCC is $63 \%$. Although our liver clinic routinely uses alpha-fetoprotein as an adjunct to imaging screening, it is acknowledged that it is neither sensitive nor specific for early diagnosis of $\mathrm{HCC}$ [8].

Once a nodule is detected, further follow-up depends on the size of the lesion(s), with both the American Association of the Society of Liver Diseases (AASLD) and the European Association for the Study of the Liver, European Organisation for Research and Treatment of Cancer (EASL-EORTC) using a threshold for further management of $1 \mathrm{~cm}$. For nodules measuring less than $1 \mathrm{~cm}$, the patient returns for a repeat ultrasound at 3 or 4 months. For nodules greater than $1 \mathrm{~cm}$, the patient undergoes a dynamic contrast enhanced computed tomography (CT) or magnetic resonance imaging (MRI). The diagnosis of $\mathrm{HCC}$ is then determined by imaging characteristics. 


\section{CT or MRI}

Unlike most other cancers, HCC can be diagnosed on imaging studies only without tissue sampling confirmation. Currently, all major consensus groups support the diagnosis of HCC with contrast-enhanced multiphasic CT, or with MRI using an extracellular contrast agent $[5,6]$. Studies have shown a similar or slightly better diagnostic performance of dynamic MR imaging compared with multiphasic CT $[9,10]$ although the difference in sensitivities is small [11-13].

The decision to perform one over the other may depend on institutional preferences, individual patient needs, and availability. Advantages of CT over MRI include lower cost, increased availability, and faster scan times. Faster scan times in particular can be an advantage in the context of a cirrhotic population with multiple morbidities and difficulty in cooperating with the breath hold requirements of MRI. Advantages of MRI include the capacity to evaluate a greater variety of tissue properties including fat content, restriction of diffusion, or T2-weighted increased signal, all of which may help in lesion detection and characterization. Lack of ionizing radiation may also be a consideration in younger patients.

\section{Ultrasound technique}

We use a standard diagnostic 3-5 Mhz linear curved array probe to evaluate the liver. Subcostal real time imaging is performed of the left lobe, followed by intercostal and subcostal views of the right lobe. Both transverse and longitudinal projections are performed. Ask the patient to adopt a left lateral decubitus position for visualization of the right lobe after initially imaging in the supine position.

Initially information on the echogenicity and coarseness of the liver echotexture is assessed, as well as smoothness or nodularity of the liver surface. Then we look for focal lesions. Comparison with prior studies is essential to assess for stability or change in small hypoechoic or hyperechoic nodules. Once a new nodule or a change in a nodule is identified the patient goes on to $\mathrm{CT}$ or MRI, often on the same day.
We look at the hepatic vasculature. Although we do not do a full Doppler evaluation of the liver, we always look at the portal vein for direction of flow with both colour and spectral techniques and for any filling defects suggestive of tumor or bland thrombus.

An interval increase in the degree of splenomegaly can indicate a worsening of portal hypertension, so we measure the spleen as a final component of the study (Table 1).

\section{MRI technique}

We perform MRI of the liver at 1.5-T field strength, although a 3.0-T field strength can also be used [14]. A phased-array coil is routinely employed. Our protocol for imaging the cirrhotic liver includes T1-weighted gradient-recalled echo (GRE) in-phase and opposedphase sequences, a moderately T2-weighted FSE sequence with an echo time of $80-90 \mathrm{msec}$, diffusion weighted imaging (DWI) and multiphase T1-weighted dynamic gadolinium-enhanced sequences.

A heavily T2-weighted sequence (echo time, $\geq 120 \mathrm{msec}$ ) can help to distinguish between cystic and solid lesions and a fast sequence, such as single-shot FSE (or halfFourier acquisition turbo spin-echo-half-Fourier rapid acquisition with relaxation enhancement), is used for this purpose.

The sequences used can vary according to vendor and personal preferences. To improve image quality, sequences should be performed during suspended respiration or should be respiratory averaged (some T2weighted sequences). Suspending respiration at end expiration produces more consistent breath holding compared with end inspiration but is more difficult for patients [15]. GRE sequences are widely used for T1weighted imaging. Using a dual gradient-echo sequence that allows simultaneous acquisition of the earliest opposed-phase and in-phase images minimizes misregistration and improves the characterization of focal lesions and diffuse liver disease [16]. The acquisition of the earliest opposed-phase echo $(2.2 \mathrm{msec}$ at $1.5-\mathrm{T}$ and $1.15 \mathrm{msec}$ at $3-\mathrm{T}$ imaging) followed by the subsequent

Table 1 Summary of imaging techniques for ultrasound, MRI and CT

\begin{tabular}{|c|c|c|}
\hline Ultrasound liver & MRI liver & $\mathrm{CT}$ liver \\
\hline $\begin{array}{l}\text { 3-5 MHz Curvilinear Probe } \\
\text { Transverse and longitudinal imaging, } \\
\text { to include supine and left lateral } \\
\text { decubitus positions } \\
\text { Doppler evaluation of the portal vein } \\
\text { Spleen measurement }\end{array}$ & $\begin{array}{l}\text { Sequences } \\
\text { Cor T2-w Single Shot Fast Spin } \\
\text { Echo +/-Fat Saturation (FS) } \\
\text { Ax T2-w Fast Spin Echo FS } \\
\text { Ax Diffusion Weighted Imaging } \\
\text { Ax dual gradient echo } \\
\text { Ax 3D Spoiled Gradient Echo FS pre and post dynamic contrast } \\
\text { enhancement (and coronal reconstruction in venous phase) } \\
\text { Ax 2D Spoiled Gradient Echo FS } \\
\text { post contrast delayed phase } \\
10 \text { ml Gadavist } \\
\text { Subtraction imaging provided }\end{array}$ & $\begin{array}{l}\text { Non contrast phase } \\
\text { IV Contrast: lohexol } 100 \mathrm{~mL} \\
\text { Bolus tracking for arterial phase (average } 30 \mathrm{~s} \text { ) } \\
\text { Venous phase } 65 \mathrm{~s} \\
\text { Delayed phase } 240 \mathrm{~s} \\
\text { Single breath for each phase } \\
\text { Injection rate min } 4 \mathrm{ml} / \mathrm{s} \\
\text { Slice thickness } 3 \mathrm{~mm} \mathrm{no} \mathrm{overlap} \\
\text { Coronal reconstructions in venous phases } \\
\text { provided. } \\
\text { Subtraction imaging optional }\end{array}$ \\
\hline
\end{tabular}


in-phase echo enables the distinction between signal intensity loss caused by the presence of lipid seen on opposed-phase images and signal intensity loss due to susceptibility artifact from hepatic iron deposition, which is exaggerated on the longer of the two echoes (usually in phase).

Three-dimensional gadolinium-enhanced GRE sequences are preferred to two-dimensional GRE sequences because of the thinner sections obtained, which improve lesion detection and permit multiplanar image reconstructions for presurgical planning [17]. Section thickness should not exceed $4 \mathrm{~mm}$ for three-dimensional sequences and $6 \mathrm{~mm}$ for two-dimensional sequences. Contrast agent bolus timing is strongly recommended, based on our experience and review of the literature [18], to ensure the consistent capturing of the arterial-dominant phase; fixed delay is not a reliable method in this patient population. Options include use of a test bolus and various automated detection methods [19]. Hypervascular HCC is most conspicuous in the late arterial phase and can be missed if the arterial-dominant phase images are acquired early [20]. A timing bolus is not essential if rapid multiphase arterial imaging is performed. To improve lesion characterization-for example, to detect washout or delayed contrast material retention of hemangioma and cholangiocarcinoma-multiphase dynamic gadoliniumenhanced imaging should include three contrastenhanced phases or more. We routinely acquire four sets of images after gadolinium-based contrast material injection in the arterial-dominant (automated timing, usually 20-35 s), venous (60-90 s), interstitial (120$150 \mathrm{~s}$ ), and delayed (5 min) phases of hepatic enhancement. The highest spatial resolution should be used without compromising signal intensity, taking into account patients' breath-holding capacity. Parallel imaging techniques can be applied to improve spatial resolution and/or reduce acquisition time. However, these techniques should be implemented with care, because they can result in image artifacts and reduced lesion conspicuity [21].

We find ourselves frequently dependent on subtraction imaging because of the intrinsic high signal demonstrated by nodules in the cirrhotic liver, including regenerative, dysplastic and malignant nodules. Intrinsic high signal can also be demonstrated in successfully treated HCC [22]. Unenhanced images can be subtracted from arterial-phase gadolinium-enhanced images to assess for arterial enhancement in nodules [23]. Subtraction can be performed if the unenhanced and gadolinium-enhanced imaging sequences are identical, if the imager is not retuned between acquisitions, and if there are no image rescaling issues. Acquiring the unenhanced and gadolinium-enhanced images in a single series rather than in separate series minimizes these differences and is possible with most systems. Patients should be instructed to hold their breath in a similar fashion during all sequences to minimize misregistration artifacts, which appear as a bright line at the edge of organs owing to incomplete overlap. At this point the ability of the MR radiographer or technician to coach the patient is crucial. Consistent breath holds are important in many MR sequences because of the lengths of the scans, but for subtraction imaging it is impossible to overemphasise the absolute requirement for good breath holds. If the patient, despite careful coaching, is unable to hold his/her breath, then CT, despite the change in modality, may be the better form of imaging.

Diffusion weighted imaging increases the detection rate of HCC, particularly for small tumours [24-26]. Bvalues typically used include one in the low range (0$50 \mathrm{~s} / \mathrm{mm} 2)$ and one in the intermediate-to-high range (400-800 s/mm2). We find that the DWI sequence frequently helps us to lean in favour or against small arterial enhancing lesions with equivocal washout as HCC, as well as assisting us in bringing our attention to small lesions which are inconspicuous on contrastenhanced sequences [27]. Tumors can be obscured on DWI because of the increased DWI signal in fibrotic liver parenchyma and subsequent decreased lesion to liver contrast [28]. In addition, DWI signal may be seen with other hepatic malignancies, such as metastases and intrahepatic cholangiocarcinomas [28-30].

Both extracellular and hepatobiliary agents can be used for imaging of the liver. We favour the use of the more expensive hepatobiliary agents only in specific cases where key decisions are to be made with regard to transplant or locoregional treatment. Indeed, hepatobiliary agents can present radiologists with greater diagnostic conundrums in contrast to more clarity.

Extracellular gadolinium-based contrast agents (for example, gadopentetate dimeglumine (Gd-DTPA), Magnevist ${ }^{\circ}$, Bayer HealthCare), distribute from the vascular space into the interstitial compartment. The standard dose is $0.1 \mathrm{mmol} / \mathrm{kg}$ typically injected intravenously at a rate of $2 \mathrm{~mL} / \mathrm{s}$ followed by a normal saline "flush" of 20 to $50 \mathrm{~mL}$.

Hepatobiliary agents distribute into the interstitial space, but, importantly for hepatic imaging, are also taken up by hepatocytes with subsequent biliary excretion. Multihance, Bracco Diagnostics, Princeton, NJ, USA) was the first to be approved. Approximately $95 \%$ of this agent is excreted by the kidney, but 3 to $5 \%$ is taken up by the normal hepatocytes and excreted into the biliary tract. Gadoxetate Disodium (U.S: Eovist, Europe: Primovist, Bayer Healthcare Pharmaceuticals, Wayne, NJ, USA) has approximately 50:50 excretion between renal (glomerular filtration) and hepatocyte uptake/biliary excretion. This can therefore be used for 
the early dynamic imaging phase in the liver, as above, followed by a $20 \mathrm{~min}$ T1-weighted imaging phase where the liver is of higher signal intensity and nonhepatocyte containing masses will be of low signal intensity. Hepatocyte-specific contrast agents have been shown in many studies to increase lesion sensitivity for HCC by capitalizing on evidence that poorly differentiated HCCs do not contain functioning hepatocytes and bile ducts, and therefore demonstrate hypointense signal relative to the surrounding liver parenchyma [30, 31]. Combining contrast-enhanced MRI features and hepatobiliary phase imaging has demonstrated sensitivities and specificities of greater than $90 \%$ [31].

Potential pitfalls that apply specifically to Eovist/Primovist include transient marked motion on arterial phase images, inability to assess washout after the portal venous phase due to early parenchymal enhancement, difficulty identifying "capsule appearance" due to hepatic parenchymal enhancement, and difficulty identifying venous tumor invasion due to more rapid venous clearance and decreased vein to liver contrast [32,33].

The use of hepatobiliary agents for the diagnosis of $\mathrm{HCC}$ is in transition. Some major HCC imaging guidelines do not mention this class contrast agents [5, 6, 34], while other societies or organizations recommend their use [35]. It remains unclear whether hepatobiliary phase contrast hypoenhancement [32] will be more widely incorporated in comparison with conventional extracellular contrast agent imaging characteristics for the diagnosis of HCC (Table 1).

\section{CT technique}

Multidetector CT (MDCT) allows fast, high-quality, thin-section imaging and permits $3 \mathrm{D}$ reconstruction with better spatial resolution than that of MRI. Fast injection rates $(4-8 \mathrm{ml} / \mathrm{s})$ provide more reliable enhancement during the hepatic arterial phase and increase the sensitivity of CT to liver lesions. Studies have demonstrated hypervascular components in $81-89 \%$ of HCCs [36]. For patients with contraindications to MRI CT serves as an adequate alternative.

CT imaging technique is based on the same principles as dynamic contrast MRI, using arterial enhancement, delayed washout, and a delayed enhancing pseudocapsule as the pillars of diagnosis. The precontrast images serve as a baseline to gauge subsequent enhancement. Following the injection of $100 \mathrm{ml}$ of Omnipaque 350 (Iohexol) we use a bolus tracking system (threshold attenuation in the aorta $150 \mathrm{HU}$ ) to initiate arterial phase breathhold imaging through the liver. Subsequent series of images are taken at $65 \mathrm{~s}$ and $240 \mathrm{~s}$ to provide venous and delayed phase imaging Subtraction images (postcontrast minus precontrast) may be helpful for detection of enhancement and evaluation of its degree [37] (Table 1).

\section{Diagnosis of HCC and report writing}

The hallmark feature of HCC on both CT and MRI is late arterial enhancement with washout relative to the liver parenchyma during the venous or delayed phases (3-5 min post injection) (Fig. 1). This pattern of enhancement has been shown to demonstrate high specificity and positive predictive value [38-40] making it the noninvasive standard for HCC diagnosis [5, 6, 35, 41-44].

In addition to the enhancement pattern, additional features of HCC have been described which are also specific for HCC including capsular enhancement [30, 45, 46]. Capsular enhancement (Fig. 2) is defined as a persistent peripheral enhancing rim seen on venous and delayed phases.

More specific to MRI, a diagnosis of HCC is often attributed to a lesion showing only arterial enhancement or only washout and pseudocapsule formation, if the lesion also demonstrates increased signal intensity on T2-weighted mages $[47,48]$ or if the lesion restricts
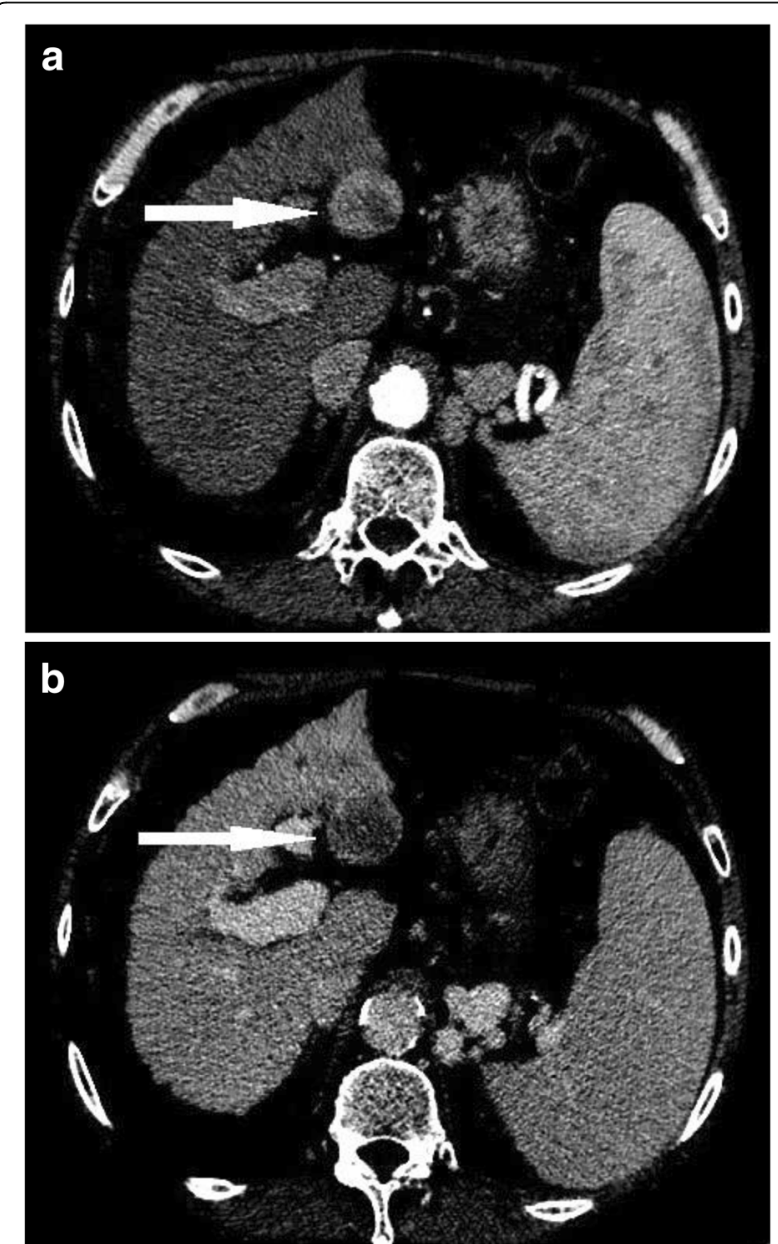

Fig. 154 year old male with hepatitis C cirrhosis. CT shows an arterial enhancing nodule a with washout of contrast in the delayed phase $\mathbf{b}$ consistent with hepatocellular carcinoma 


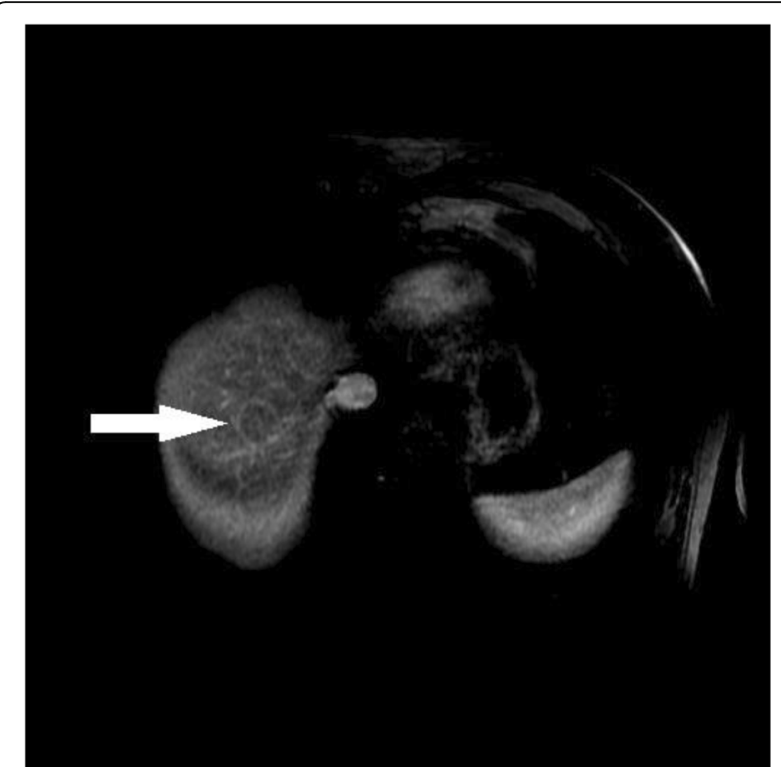

Fig. 267 year old male with alchohol liver disease and cirrhosis Venous phase MRI with gadolinium demonstrates an HCC nodule at the dome of the liver with capsular enhancement

diffusion [25, 27, 49], although some caution should be applied to both of these adjuncts as they can result in false positive interpretations [50] (Table 2).

Intracellular lipid detected within a nodule on dual-echo in and opposed phase T1-weighted MRI is an additional finding which has been shown to be reasonably specific for HCC. This can be a useful addition to the toolbox when looking at a lesion with non-specific enhancement characteristics as intracellular lipid is very rare in a regenerative or dysplastic nodules [51] (Fig. 3).

In the event of uncertainty a consensus opinion is reached from the available liver imaging specialists in the department. Lesions with focal hepatic arterial enhancement, but without washout, capsule enhancement, or abnormal increased T2 signal, are considered dysplastic nodules (if clearly a defined nodule) or nonspecific hypervascular lesions (if nonmarginated and subcapsular).

We review prior imaging and clinical information for all patients. An understanding of the treatment options for HCC under the current guidelines is essential. We structure the conclusions of our reports so that the

Table 2 MRI major and ancillary features for the diagnosis of HCC

\begin{tabular}{ll}
\hline HCC: major feature $s$ & HCC: ancillary features \\
\hline Arterial phase enhancement & T2-w hyperintensity \\
Delayed phase "washout" & Restriction of diffusion \\
Threshold growth & Intra-lesional fat \\
Delayed enhancing capsule & \\
\hline
\end{tabular}
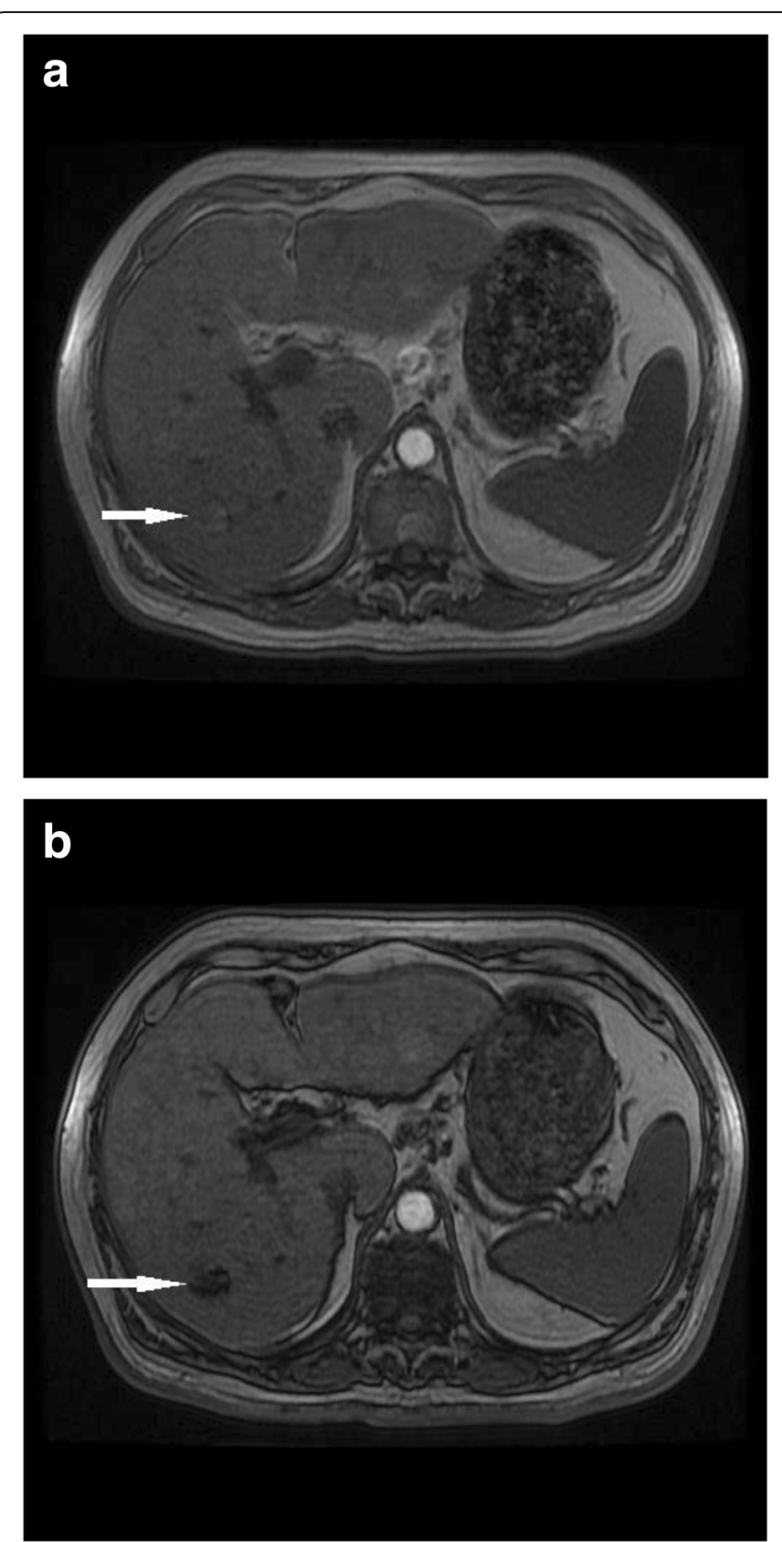

Fig. 371 year old male with hepatitis C cirrhosis. Signal drop out on opposed phase imaging (b) in comparison with in phase imaging (a). The findings represent intracellular lipid in an HCC tumour

multidisciplinary liver group can make informed decisions in the context of the options available.

Reports indicate the size (largest axial or coronal section diameter), number, and location of HCC lesions. The Couinaud classification is used for anatomic reference [52]. Although the system was designed for surgical planning it is universally accepted, simple, and more concise than the descriptive terms for the segmental anatomy. The coronal measurement is frequently omitted in reports but is important because it affects the treatment stratification, both for transplant evaluation and for determination of the type of locoregional therapy to be used. 
We number the tumors from 1 to 4 . If there are more than 4 lesions then we determine whether there is unilobar or bilobar disease and describe how many lesions there are in each lobe, again numbering them so that they can be easily detected. We believe in the importance of providing series and image numbers for each lesion up to 4 lesions so that if the reporting radiologist is not present at the multidisciplinary meeting, or if surgeons or liver specialists are looking at the images, they can find the lesions quickly and not become confused by other confounding imaging findings.

For each lesion the T1-weighted, T2-weighted, diffusion weighted and contrast enhanced characteristics are always described. If there are ancillary findings, for example signal dropout on opposed phase imaging in contrast with in phase imaging, then we add those as well. Although we do not strictly apply a LIRADS (Liver Imaging Reporting and Data System) number to each lesion, we report findings in the context of the LIRADS criteria as these are the current most comprehensive guidelines used to stratify the risk malignancy in the context of cirrhosis and HCC [53]. LIRADS is a useful system to use when there is not close communication in a multidisciplinary setting. It is easily accessible online and the system is helpful for those cases where there is some uncertainty.

For specific examples which are not clearly covered by guidelines, our experiences are that small nodule-like arterial enhancing lesions which do not show associated washout, but which increase in conspicuity over time, merit close attention on follow up imaging as these often develop ancillary features of washout, pseudocapsule or restricted diffusion over time. Small foci of restricted diffusion or high T2-weighted signal with arterial enhancement often turn out to be HCC, whereas small foci or restricted diffusion without arterial enhancement, and without other ancillary features, are very common, and are almost always not related to cancer.

A review for extrahepatic disease is essential as metastatic disease changes all of the treatment pathways. The lungs should be imaged once HCC is diagnosed. Metastatic disease is seen in multiple locations but portal lymph nodes, peritoneum, adrenal glands and bones are the more frequent locations.

\section{Selection and staging}

Once a patient is diagnosed with HCC, a multidisciplinary approach is adopted to determine optimal therapy and further management. Our group includes transplant surgeons, hepatologists, oncologists, radiation oncologists, and cross-sectional and interventional radiologistsWe prepare the cases for presentation each week.

Although several staging schema have been developed, none have been universally adopted. A few main factors have been identified as influential in the prognosis of patients with HCC. These include liver function, tumor size and number, tumor extent, including vascular invasion and extrahepatic spread, evidence of portal hypertension, and clinical performance status. Tumor proximity to large vessels and main bile ducts can also be pertinent with regard to ablative therapies, and is worth mentioning if these treatments are likely to be considered.

CT and MRI are useful in identifying tumor extent and extrahepatic spread. They also provide secondary evidence of portal hypertension, including the presence of splenomegaly and portosystemic collaterals. Imaging of the chest is also recommended as part of the initial work up, given that lung and bone are common sites for HCC metastasis. A bone scan can also be performed if there is a suspicion for osseous metastasis, or if the patient is being considered for liver transplantation.

The Barcelona Clinic Liver Cancer (BCLC) system links the staging of HCC in patients with cirrhosis with treatment options, making it the most commonly adopted staging system $[5,6]$.

The BCLC system identifies those patients with early stage $\mathrm{HCC}$ who may benefit from curative therapies (stage 0 and $\mathrm{A}$ ), those at intermediate (stage B) or advanced (stage $C$ ) stages who may benefit from palliative treatments, and those who are most suitable for best supportive care (stage D). Curative treatment options, including transplantation, resection, and ablation for patients with early stage disease depends on local factors, patient specific issues, and patient preference. Palliative, non-curative treatment options include transcatheter arterial chemoembolization (TACE) for stage B disease, radioembolisation, and sorafenib for advanced stage $\mathrm{C}$ disease. TACE is also increasingly used as a "bridge" to transplant, and in some cases to downstage patients so that they can become candidates for a transplant list $[54,55]$.

In equivocal cases where the diagnosis of HCC is uncertain in small lesions, a reasonable approach is to wait 3 months and image again $[56,57]$.

\section{Post therapy imaging}

Because many patients with HCC do not meet criteria for transplantation or surgery, a large proportion of patients receive locoregional therapy or systemic therapy and therefore require post-therapy imaging to evaluate for initial response and recurrent disease. No established guidelines for ideal surveillance time intervals exist. Recurrence is 6.5 times more likely to occur in the first year after therapy than in the second year, so most guidelines suggest 3 monthly interval imaging in the first year after treatment [58]. We follow up with imaging at 3 month intervals for one year followed by 6 month intervals for 2 years, and then we return to ultrasound screening. It is important to use the same modality for each follow up as comparison between CT and MRI can 

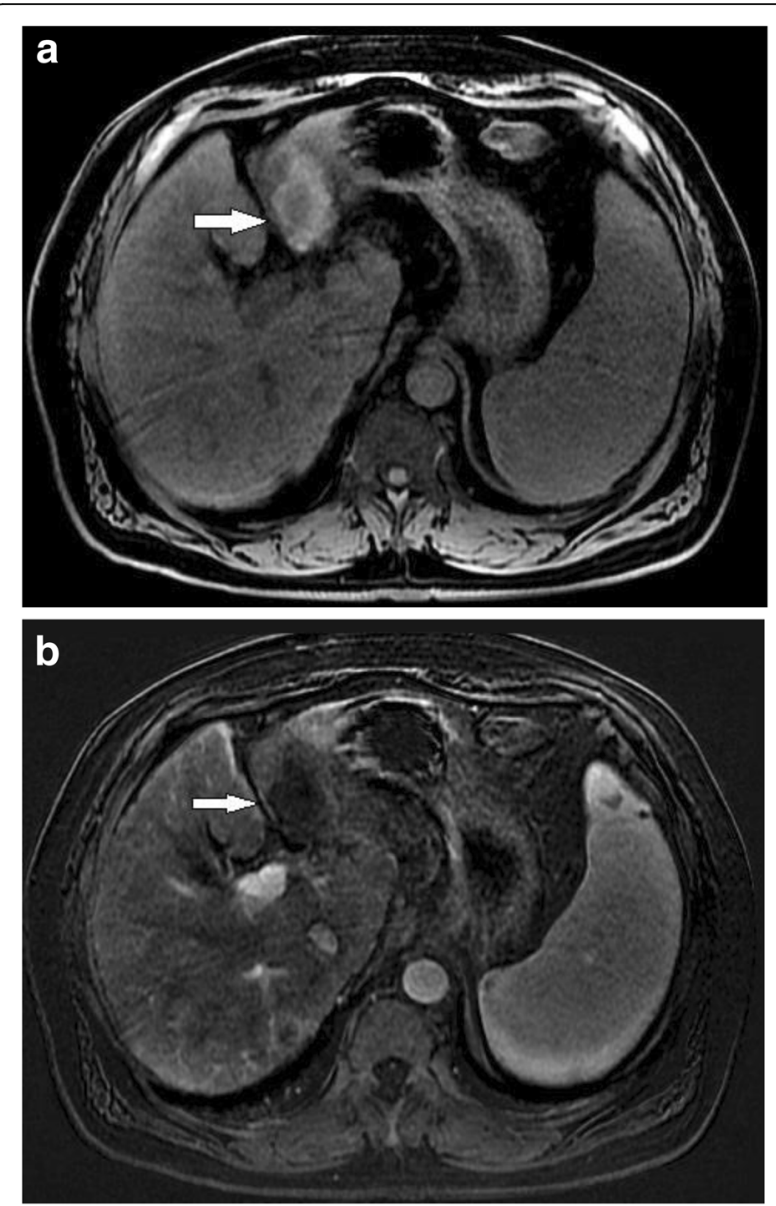

Fig. 466 year old female with hepatitis C cirrhosis post microwave ablation of HCC Precontrast image post microwave ablation (a) show a cavity with intrinsic high signal on T1 weighted imaging. A subtraction image $(\mathbf{b})$ removes the high signal resulting in no evidence of enhancement

be challenging. We generally use MRI for follow up as the imaging findings can be more difficult to interpret following treatment and the subtraction images can be really useful (Fig. 4).

Several systems have been developed to objectively evaluate the response of $\mathrm{HCC}$ to locoregional therapy. Some of these are based on tumor size, such as the WHO (World Health Organisation) and RECIST (Response Evaluation Criteria in Solid Tumours) criteria [59, 60],

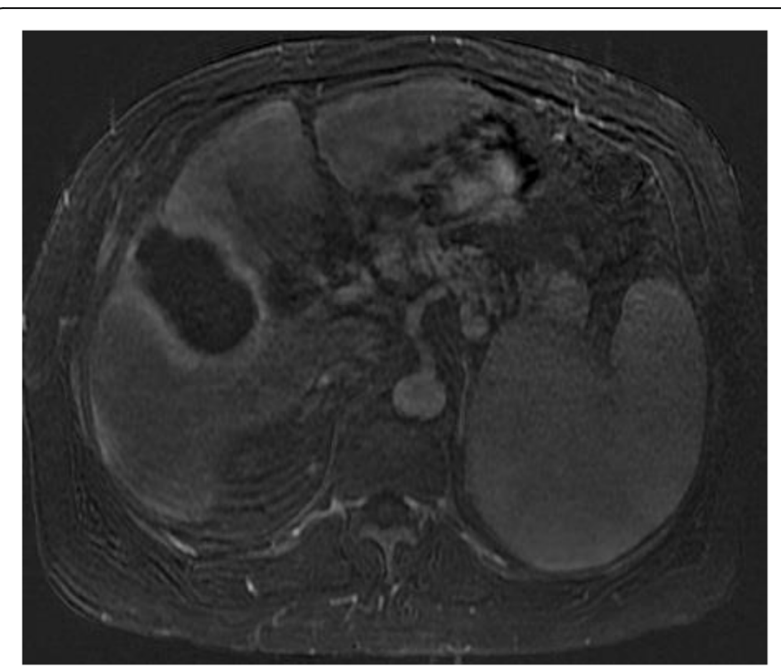

Fig. 563 year old male with cirrhosis and HCC treated with microwave ablation. A thin rim of enhancement post ablation, consistent with hyperemia adjacent to the ablation zone, is a normal finding and does not represent recurrent tumour

while others, such as EASL, AASLD, and mRECIST, are based on the assessment of residual enhancing HCC [61, 62]. mRECIST, or modified RECIST, therefore does not evaluate tumour bulk itself, as does RECIST, as this may not change after treatment, or may even increase, but assesses the volume of residual functional tumour or arterial enhancing tissue [63]. Studies have shown that the mRECIST and EASL enhancement-based protocols correlate more accurately with residual disease burden and with survival after therapy than the size-based protocols for patients treated with ablation, radioembolization and TACE [63-67]. At our multidisciplinary meetings we use a combination of mRECIST and EASL criteria to quantify residual or recurrent tumour, along with informed discussion from the team members (Table 3).

Prior to reporting we make sure we have established the procedures performed or therapies used, as lack of awareness of these can lead to embarrassing errors in reporting. Regardless of the therapy performed, treated tumor should demonstrate an absence of enhancement. A thin rim of enhancement can be seen as a normal finding after ablation and TACE due to adjacent hyperemia and fibrosis (Fig. 5). However, residual or

Table 3 Summary of mRECIST and EASL responses

\begin{tabular}{|c|c|c|}
\hline & mRECIST & EASL \\
\hline Complete Response & $\begin{array}{l}\text { Disappearance of any intratumoral arterial enhancement } \\
\text { in all target lesions (up to } 2 \text { measurable liver lesions) }\end{array}$ & $\begin{array}{l}\text { Disappearance of any intratumoral arterial enhancement } \\
\text { in all measurable arterial enhancing liver lesions }\end{array}$ \\
\hline Partial Response & Decrease $>30 \%$ in the sum of longest diameters of viable target lesions & $\begin{array}{l}\text { Decrease }>50 \% \text { in the sum of the product of } \\
\text { bidimensional diameters of viable target lesions }\end{array}$ \\
\hline Progressive Disease & Increase $>20 \%$ in the sum of longest diameters of viable target lesions & $\begin{array}{l}\text { Increase }>25 \% \text { in the sum of the diameters of } \\
\text { viable target lesions }\end{array}$ \\
\hline Stable Disease & None of the above & None of the above \\
\hline
\end{tabular}


recurrent disease presents as thick or nodular peripheral arterial enhancement [65, 68, 69] (Fig. 6). Post-ablation changes are similar regardless of what type of ablation is performed. The ablation zone should be larger than the original tumor by between 5 and $10 \mathrm{~mm}$. If it is not, then careful attention to subtle enhancing lesions is needed. Ablation zones can decrease in size with time. An ablation zone can demonstrate high signal intensity on pre-contrast T1-weighted images as a result of coagulative necrosis, making evaluation for arterial enhancement difficult in the absence of subtraction imaging. Subtractions should therefore be routinely included within the MRI protocol [22].
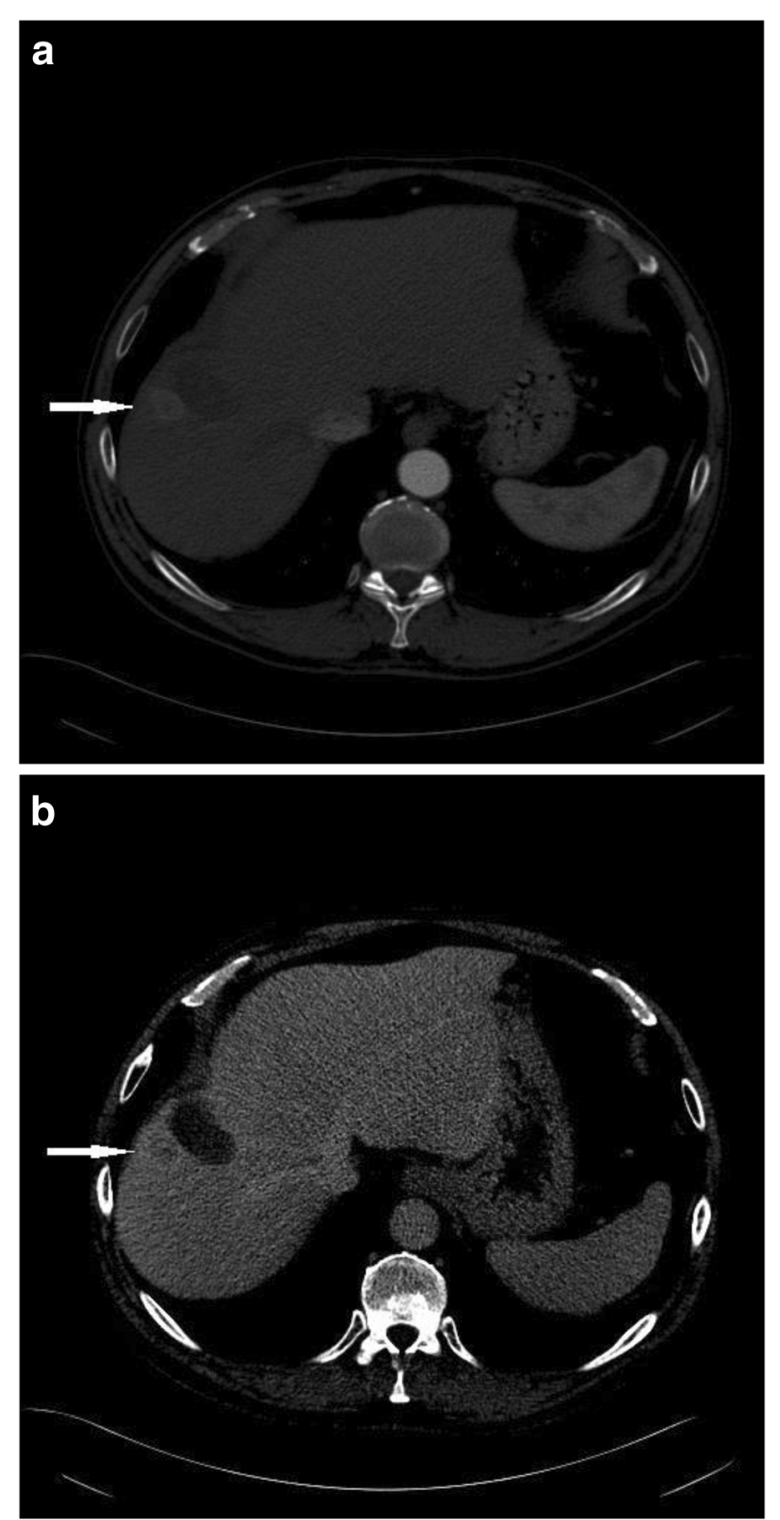

Fig. 657 year old female with cirrhosis and HCC treated with RFA. $\mathrm{CT}$ in arterial (a) and venous (b) phases shows enhancement and washout of a nodule adjacent to an RFA ablation zone

\section{Conclusions}

The accepted modality for hepatocellular carcinoma screening is ultrasound. Once HCC is suspected then CT or MRI may be used to confirm the diagnosis and establish the tumor burden for staging purposes. The BCLC classification system is the most frequently used for treatment planning. However, multidisciplinary meeting and planning is essential to ensure that the correct pathways are adopted within the context of each institution. Following surgical, locoregional, chemotherapeutic or radiotherapeutic treatment, follow up imaging and regular multidisciplinary discussion is adopted.

\section{Abbreviations}

AASLD: American Association for the Study of Liver Diseases; BCLC: Barcelona Clinic Liver Cancer; CT: Computed tomography; DWI: Diffusion weighted imaging; EASL-EORTC: European Association for the Study of the Liver, European Organisation for Research and Treatment of Cancer; FSE: HalfFourier acquisition turbo spin-echo; Gd: Gadolinium; GRE: Gradient echo; HCC: Hepatocellular carcinoma; LIRADS: Liver Image and Reporting Data System; MDCT: Multidetector computed tomography; mRECIST: modified Response Evaluation Criteria in Solid Tumours; MRI: Magnetic resonance imaging; RECIST: Response Evaluation Criteria in Solid Tumours; TACE: Transcatheter arterial chemoembolization; WHO: World Health Organisation

\section{Acknowledgements}

Not applicable.

\section{Funding}

Not applicable.

Availability of data and materials

Data sharing not applicable to this article as no datasets were generated or analysed during the current study.

\section{Authors' contributions}

JW was primary author and prepared the manuscript for publication. JR drafted the original manuscript. SA reviewed and helped edit the manuscript and provided help with images. ND reviewed, provided expert opinion and edits on treatments for HCC, and helped edit the manuscript. FS reviewed and helped edit the manuscript as an independent reviewer of the structure and content of the article. All authors read and approved the final manuscript.

\section{Competing interests}

The authors declare that they have no competing interests.

\section{Consent for publication}

Not applicable.

Ethics approval and consent to participate Not applicable.

Received: 9 December 2016 Accepted: 14 February 2017

Published online: 04 March 2017

\section{References}

1. Torre LA, Bray F, Siegel RL, Ferlay J, Lortet-Tieulent J, Jemal A. Global cancer statistics, 2012. CA Cancer J Clin. 2015;65(2):87-108.

2. Perz JF, Armstrong GL, Farrington LA, Hutin YJ, Bell BP. The contributions of hepatitis $B$ virus and hepatitis $C$ virus infections to cirrhosis and primary liver cancer worldwide. J Hepatol. 2006;45(4):529-38.

3. Bugianesi E. EASL-EASD-EASO Clinical Practice Guidelines for the management of non-alcoholic fatty liver disease: disease mongering or call to action? Diabetologia. 2016;59(6):1145-7.

4. Seeff LB. Introduction: The burden of hepatocellular carcinoma. Gastroenterology. 2004;127(5 Suppl 1):S1-4.

5. Bruix J, Sherman M. Management of hepatocellular carcinoma: an update. Hepatology. 2011;53(3):1020-2. 
6. EASL-EORTC clinical practice guidelines: management of hepatocellular carcinoma. J Hepatol. 2012;56(4):908-43.

7. Singal A, Volk ML, Waljee A, et al. Meta-analysis: surveillance with ultrasound for early-stage hepatocellular carcinoma in patients with cirrhosis. Aliment Pharmacol Ther. 2009;30(1):37-47.

8. Lok AS, Sterling RK, Everhart JE, et al. Des-gamma-carboxy prothrombin and alpha-fetoprotein as biomarkers for the early detection of hepatocellular carcinoma. Gastroenterology. 2010;138(2):493-502.

9. Choi TW, Lee JM, Kim JH, Yu MH, Han JK, Choi Bl. Comparison of multidetector CT and gadobutrol-enhanced MR imaging for evaluation of small, solid pancreatic lesions. Korean J Radiol. 2016;17(4):509-21.

10. Guo J, Seo Y, Ren S, et al. Diagnostic performance of contrast-enhanced multidetector computed tomography and gadoxetic acid disodium-enhanced magnetic resonance imaging in detecting hepatocellular carcinoma: direct comparison and a meta-analysis. Abdom Radiol. 2016:41(10):1960-72.

11. Burrel M, Llovet JM, Ayuso C, et al. MRI angiography is superior to helical CT for detection of HCC prior to liver transplantation: an explant correlation. Hepatology. 2003;38(4):1034-42.

12. Kim YK, Kim CS, Chung GH, et al. Comparison of gadobenate dimeglumineenhanced dynamic MRI and 16-MDCT for the detection of hepatocellular carcinoma. AJR Am J Roentgenol. 2006;186(1):149-57.

13. Lim JH, Kim CK, Lee WJ, et al. Detection of hepatocellular carcinomas and dysplastic nodules in cirrhotic livers: accuracy of helical $C T$ in transplant patients. AJR Am J Roentgenol. 2000;175(3):693-8.

14. Hussain SM, Wielopolski PA, Martin DR. Abdominal magnetic resonance imaging at 3.0 T: problem or a promise for the future? Top Magn Reson Imaging. 2005;16(4):325-35.

15. Kimura T, Hirokawa Y, Murakami Y, et al. Reproducibility of organ position using voluntary breath-hold method with spirometer for extracranial stereotactic radiotherapy. Int J Radiat Oncol Biol Phys. 2004;60(4):1307-13.

16. Merkle EM, Nelson RC. Dual gradient-echo in-phase and opposed-phase hepatic MR imaging: a useful tool for evaluating more than fatty infiltration or fatty sparing. Radiographics. 2006;26(5):1409-18.

17. Lee VS, Lavelle MT, Rofsky NM, et al. Hepatic MR imaging with a dynamic contrast-enhanced isotropic volumetric interpolated breath-hold examination: feasibility, reproducibility, and technical quality. Radiology. 2000;215(2):365-72.

18. Sharma P, Kitajima HD, Kalb B, Martin DR. Gadolinium-enhanced imaging of liver tumors and manifestations of hepatitis: pharmacodynamic and technical considerations. Top Magn Reson Imaging. 2009;20(2):71-8.

19. Hussain HK, Londy FJ, Francis IR, et al. Hepatic arterial phase MR imaging with automated bolus-detection three-dimensional fast gradient-recalled-echo sequence: comparison with test-bolus method. Radiology. 2003;226(2):558-66.

20. Mori K, Yoshioka H, Takahashi N, et al. Triple arterial phase dynamic MRI with sensitivity encoding for hypervascular hepatocellular carcinoma: comparison of the diagnostic accuracy among the early, middle, late, and whole triple arterial phase imaging. AJR Am J Roentgenol. 2005;184(1):63-9.

21. Vogt FM, Antoch $G$, Hunold P, et al. Parallel acquisition techniques for accelerated volumetric interpolated breath-hold examination magnetic resonance imaging of the upper abdomen: assessment of image quality and lesion conspicuity. J Magn Reson Imaging. 2005;21(4):376-82.

22. Winters SD, Jackson S, Armstrong GA, Birchall IW, Lee KH, Low G. Value of subtraction MRI in assessing treatment response following imageguided loco-regional therapies for hepatocellular carcinoma. Clin Radiol. 2012:67(7):649-55

23. Yu JS, Kim YH, Rofsky NM. Dynamic subtraction magnetic resonance imaging of cirrhotic liver: assessment of high signal intensity lesions on nonenhanced T1-weighted images. J Comput Assist Tomogr. 2005;29(1):51-8.

24. Park MS, Kim S, Patel J, et al. Hepatocellular carcinoma: detection with diffusion-weighted versus contrast-enhanced magnetic resonance imaging in pretransplant patients. Hepatology. 2012;56(1):140-8.

25. Xu PJ, Yan FH, Wang JH, Shan Y, Ji Y, Chen CZ. Contribution of diffusionweighted magnetic resonance imaging in the characterization of hepatocellular carcinomas and dysplastic nodules in cirrhotic liver. J Comput Assist Tomogr. 2010;34(4):506-12.

26. Le Moigne F, Durieux M, Bancel B, et al. Impact of diffusion-weighted MR imaging on the characterization of small hepatocellular carcinoma in the cirrhotic liver. Magn Reson Imaging. 2012;30(5):656-65.

27. Wu LM, Xu JR, Lu Q, Hua J, Chen J, Hu J. A pooled analysis of diffusionweighted imaging in the diagnosis of hepatocellular carcinoma in chronic liver diseases. J Gastroenterol Hepatol. 2013;28(2):227-34.
28. Park MJ, Kim YK, Lee MW, et al. Small hepatocellular carcinomas: improved sensitivity by combining gadoxetic acid-enhanced and diffusion-weighted MR imaging patterns. Radiology. 2012;264(3):761-70.

29. Miller FH, Hammond N, Siddiqi AJ, et al. Utility of diffusion-weighted MRI in distinguishing benign and malignant hepatic lesions. J Magn Reson Imaging. 2010;32(1):138-47.

30. Rhee H, Kim MJ, Park MS, Kim KA. Differentiation of early hepatocellular carcinoma from benign hepatocellular nodules on gadoxetic acid-enhanced MRI. Br J Radiol. 2012;85(1018):e837-44.

31. Liu X, Zou L, Liu F, Zhou Y, Song B. Gadoxetic acid disodium-enhanced magnetic resonance imaging for the detection of hepatocellular carcinoma: a meta-analysis. PLoS One. 2013;8(8):e70896.

32. Motosugi U, Bannas P, Sano K, Reeder SB. Hepatobiliary MR contrast agents in hypovascular hepatocellular carcinoma. J Magn Reson Imaging. 2015:41(2):251-65

33. Hope TA, Fowler $\mathrm{K}$, Sirlin $\mathrm{CB}$, et al. Hepatobiliary agents and their role in LI-RADS. Abdom Imaging. 2015:40(3):613-25.

34. Wald C, Russo MW, Heimbach JK, Hussain HK, Pomfret EA, Bruix J. New OPTN/UNOS policy for liver transplant allocation: standardization of liver imaging, diagnosis, classification, and reporting of hepatocellular carcinoma. Radiology. 2013;266(2):376-82

35. Kudo M, Izumi N, Kokudo $\mathrm{N}$, et al. Management of hepatocellular carcinoma in Japan: consensus-based clinical practice guidelines proposed by the Japan Society of Hepatology (JSH) 2010 updated version. Dig Dis. 2011;29(3):339-64.

36. Murakami T, Kim T, Takamura M, et al. Hypervascular hepatocellular carcinoma: detection with double arterial phase multi-detector row helical CT. Radiology. 2001;218(3):763-7.

37. Miraglia R, Pietrosi G, Maruzzelli $L$, et al. Predictive factors of tumor response to trans-catheter treatment in cirrhotic patients with hepatocellular carcinoma: a multivariate analysis of pre-treatment findings. World J Gastroenterol. 2007:13(45):6022-6

38. Forner A, Vilana R, Ayuso C, et al. Diagnosis of hepatic nodules $20 \mathrm{~mm}$ or smaller in cirrhosis: Prospective validation of the noninvasive diagnostic criteria for hepatocellular carcinoma. Hepatology. 2008;47(1):97-104.

39. Kim SE, Lee HC, Shim JH, et al. Noninvasive diagnostic criteria for hepatocellular carcinoma in hepatic masses $>2 \mathrm{~cm}$ in a hepatitis B virus-endemic area. Liver Int. 2011:31(10):1468-76.

40. Leoni S, Piscaglia F, Golfieri R, et al. The impact of vascular and nonvascular findings on the noninvasive diagnosis of small hepatocellular carcinoma based on the EASL and AASLD criteria. Am J Gastroenterol. 2010;105(3):599-609.

41. Benson 3rd AB, Abrams TA, Ben-Josef E, et al. NCCN clinical practice guidelines in oncology: hepatobiliary cancers. J Natl Compr Canc Netw. 2009;7(4):350-91.

42. Omata M, Lesmana LA, Tateishi $R$, et al. Asian Pacific Association for the Study of the Liver consensus recommendations on hepatocellular carcinoma. Hepatol Int. 2010;4(2):439-74.

43. Verslype C, Rosmorduc O, Rougier P. Hepatocellular carcinoma: ESMOESDO Clinical Practice Guidelines for diagnosis, treatment and follow-up. Ann Oncol. 2012;23 Suppl 7:vii41-8.

44. Santillan CS, Tang A, Cruite I, Shah A, Sirlin CB. Understanding LI-RADS: a primer for practical use. Magn Reson Imaging Clin N Am. 2014;22(3):337-52.

45. Khan AS, Hussain HK, Johnson TD, Weadock WJ, Pelletier SJ, Marrero JA. Value of delayed hypointensity and delayed enhancing rim in magnetic resonance imaging diagnosis of small hepatocellular carcinoma in the cirrhotic liver. J Magn Reson Imaging. 2010;32(2):360-6.

46. Cruite I, Santillan C, Mamidipalli A, Shah A, Tang A, Sirlin CB. Liver imaging reporting and data system: review of ancillary imaging features. Semin Roentgenol. 2016;51(4):301-7.

47. Coenegrachts K, Delanote J, Ter Beek L, et al. Improved focal liver lesion detection: comparison of single-shot diffusion-weighted echoplanar and singleshot T2 weighted turbo spin echo techniques. Br J Radiol. 2007;80(955):524-31.

48. Parikh T, Drew SJ, Lee VS, et al. Focal liver lesion detection and characterization with diffusion-weighted MR imaging: comparison with standard breath-hold T2-weighted imaging. Radiology. 2008;246(3):812-22.

49. Piana G, Trinquart L, Meskine N, Barrau V, Beers BV, Vilgrain V. New MR imaging criteria with a diffusion-weighted sequence for the diagnosis of hepatocellular carcinoma in chronic liver diseases. J Hepatol. 2011;55(1):126-32.

50. Hicks RM, Yee J, Ohliger MA, et al. Comparison of diffusion-weighted imaging and T2-weighted single shot fast spin-echo: Implications for LIRADS characterization of hepatocellular carcinoma. Magn Reson Imaging. 2016;34(7):915-21. 
51. Sano K, Ichikawa T, Motosugi U, et al. Imaging study of early hepatocellular carcinoma: usefulness of gadoxetic acid-enhanced MR imaging. Radiology. 2011;261(3):834-44.

52. Couinaud C. Liver lobes and segments: notes on the anatomical architecture and surgery of the liver. Presse Med. 1954;62(33):709-12.

53. ACo. R. Liver imaging reporting and data system version. American College of Radiology: https://nrdr.acr.org/lirads/. Accessed 10 Dec 2016.

54. Prasad MA, Kulik LM. The role of bridge therapy prior to orthotopic liver transplantation. J Natl Compr Canc Netw. 2014;12(8):1183-90. quiz 91.

55. San Miguel C, Muffak K, Triguero J, et al. Role of transarterial chemoembolization to downstage hepatocellular carcinoma within the Milan criteria. Transplant Proc. 2015;47(9):2631-3.

56. An C, Choi YA, Choi D, et al. Growth rate of early-stage hepatocellular carcinoma in patients with chronic liver disease. Clin Mol Hepatol. 2015;21(3):279-86.

57. Jha RC, Zanello PA, Nguyen XM, et al. Small hepatocellular carcinoma: MRI findings for predicting tumor growth rates. Acad Radiol. 2014;21(11):1455-64.

58. Boas FE, Do B, Louie JD, et al. Optimal imaging surveillance schedules after liver-directed therapy for hepatocellular carcinoma. J Vasc Interv Radiol. 2015;26(1):69-73.

59. Bogaerts J, Ford R, Sargent D, et al. Individual patient data analysis to assess modifications to the RECIST criteria. Eur J Cancer. 2009;45(2):248-60.

60. Miller AB, Hoogstraten B, Staquet M, Winkler A. Reporting results of cancer treatment. Cancer. 1981;47(1):207-14.

61. Lencioni R. New data supporting modified RECIST (mRECIST) for Hepatocellular Carcinoma. Clin Cancer Res. 2013;19(6):1312-4.

62. Lencioni R, Llovet JM. Modified RECIST (mRECIST) assessment for hepatocellular carcinoma. Semin Liver Dis. 2010;30(1):52-60.

63. Gillmore R, Stuart S, Kirkwood A, et al. EASL and mRECIST responses are independent prognostic factors for survival in hepatocellular cancer patients treated with transarterial embolization. J Hepatol. 2011;55(6):1309-16.

64. Bargellini I, Bozzi E, Campani D, et al. Modified RECIST to assess tumor response after transarterial chemoembolization of hepatocellular carcinoma: $\subset$ Tpathologic correlation in 178 liver explants. Eur J Radiol. 2013;82(5):e212-8.

65. Forner A, Ayuso C, Varela M, et al. Evaluation of tumor response after locoregional therapies in hepatocellular carcinoma: are response evaluation criteria in solid tumors reliable? Cancer. 2009;115(3):616-23.

66. Riaz A, Memon K, Miller FH, et al. Role of the EASL, RECIST, and WHO response guidelines alone or in combination for hepatocellular carcinoma: radiologic-pathologic correlation. J Hepatol. 2011;54(4):695-704.

67. Yeo DM, Choi JI, Lee YJ, Park MY, Chun HJ, Lee HG. Comparison of RECIST, mRECIST, and choi criteria for early response evaluation of hepatocellular carcinoma after transarterial chemoembolization using drug-eluting beads. J Comput Assist Tomogr. 2014;38(3):391-7.

68. Kloeckner R, Otto G, Biesterfeld S, Oberholzer K, Dueber C, Pitton MB. $M D C T$ versus MRI assessment of tumor response after transarterial chemoembolization for the treatment of hepatocellular carcinoma. Cardiovasc Intervent Radiol. 2010;33(3):532-40.

69. Sainani NI, Gervais DA, Mueller PR, Arellano RS. Imaging after percutaneous radiofrequency ablation of hepatic tumors: part 2, abnormal findings. AJR Am J Roentgenol. 2013;200(1):194-204.

\section{Submit your next manuscript to BioMed Central and we will help you at every step:}

- We accept pre-submission inquiries

- Our selector tool helps you to find the most relevant journal

- We provide round the clock customer support

- Convenient online submission

- Thorough peer review

- Inclusion in PubMed and all major indexing services

- Maximum visibility for your research

Submit your manuscript at www.biomedcentral.com/submit

) Biomed Central 